ARCHIVO ESPAÑOL DE ARTE, LXXIX, 314

ABRIL-JUNIO, pp. 157-168, 2006

ISSN: 0004-0428

\title{
UN CONTRATO DE LOS HERNANDOS PARA LA CAPILLA DE LES FEBRES DE LA SEO DE XÀTIVA EN 1511
}

\author{
POR \\ MERCEDES GÓMEZ-FERRER Y JUAN CORBALÁN DE CELIS \\ Departamento de Historia del Arte de la Universitat de València
}

\begin{abstract}
Se presenta el contrato firmado en 1511 por Fernando Llanos y Fernando Yañez de la Almedina, pintores castellanos conocidos como los Hernandos, para la finalización de un retablo para la capilla de la Virgen de les Febres, fundada en 1497 por D. Francisco de Borja, obispo de Teano y cardenal de Cosenza, en la colegiata de Xàtiva (Valencia). A partir del documento, se plantean una serie de hipótesis sobre la trayectoria y obra de los Hernandos tras la realización de las puertas del retablo mayor de la catedral de Valencia.

Palabras clave: Pintura renacentista, retablo, siglo XVI, Fernando Yáñez de la Almedina, Fernando Llanos, Hernandos, Francisco de Borja, colegiata de Xàtiva (Valencia)

The contract dated 1511 signed by Fernando Llanos and Fernando Yáñez de la Almedina, Castillian painters known as "the Hernandos," for the termination of an altarpiece in the chapel of the "Virgen de les Febres" is presented here. This chapel was founded in 1497 in the collegiate church of Jàtiva (Valencia) by Francisco de Borja, Bishop of Teano and Cardinal of Cosenza. Based on this document, a series of hypotheses are put forth concerning the trajectory and works of "the Hernandos" following their execution of the doors of the main altarpiece of the Cathedral of Valencia.

Key words: Renaissance painting, Altarpiece, 16th century, Fernando Yáñez de la Almedina, Fernando Llanos, The Hernandos, Francisco de Borja, Collegiate church of Jàtiva (Valencia).
\end{abstract}

\section{La capilla de les Febres de la Colegiata de Xàtiva}

La colegiata de Xàtiva (Valencia), con una azarosa historia, plagada de proyectos ambiciosos, muchos frustrados o incompletos, reconstrucciones y demoliciones, es un edificio que escapa a los análisis convencionales. Xàtiva fue una de las poblaciones importantes del territorio valenciano donde se mantuvo más tiempo la primitiva construcción musulmana y la mezquita se siguió utilizando hasta finales del siglo XVI con el añadido de capillas y altares cristianos que la fueron transformando paulatinamente. No obstante, en 1563, el cronista Viciana aún describía como "La 
yglesia mayor de la ciudad fue la mezquita de los Algarenos y fabricada por ellos, es VII naves casi cuadrangular"1. En 1596 se puso la primera piedra de lo que sería la nueva colegiata, pero muchas de sus capillas no se terminaron de demoler hasta fines del siglo XVIII. Dos de las más importantes estaban vinculadas a la familia Borja, la de santa Ana, que mandó edificar el obispo Alfonso de Borja, hacia 1450, antes de su nombramiento como pontífice y la de les Febres, costeada por Francisco de Borja, obispo de Teano en 1497. Una lápida, conservada actualmente en una de las capillas de la girola, conmemora su fundación (fig.1):

\section{"FRANCISCUS DE BORJA EPISCOPUS TEANENSIS ALEXANDRI VI PONT(IFEX) MAX(IMUS) THESAURARIUS CAMERAE APOSTOLICAE PRAESI- DENS HANC CAPELLAM SUAE DOMUS MONUMENTUM MARIAE VIRGINI DE FEBRIBUS QUATORQUE ECCLESIAE DOCTORIBUS PIE EREXIT DICA- VITQ EX VOTO ANNO SALUTIS MCCCCLXXXXVII"2.}

Francisco de Borja fue obispo de Teano, en Campania, Tesorero de Alejandro VI, presidente de la Cámara Apostólica, y cardenal de Cosenza, en Calabria, por nombramiento en 1500, que no menciona la lápida al ser posterior a la fecha de la fundación de la capilla (fig. 2). Aunque su grado de parentesco con los papas Borja no es del todo conocido, era al parecer, hijo de Martín de Borja, hermano de Rodrigo Gil de Borja, abuelo de Alejandro VI ${ }^{3}$. En ocasiones su filiación ${ }^{4}$ se ha confundido con la de otros miembros homónimos de la extensa familia Borja y con alguno de sus descendientes, especialmente un sobrino del mismo nombre y también obispo de Teano, por lo que durante un tiempo se fundieron ambas personalidades, hasta que fueron definitivamente deslindadas ${ }^{5}$.

Francisco de Borja tuvo una hermana, Francisca que casaría con Felipe Cardador quienes a su vez tuvieron dos hijos, Isabel, casada con Baltasar Ferran, y un varón a quien pusieron por nombre Francisco. Este segundo Francisco de Borja y Cardador siguió la carrera eclesiástica, residiendo en Valencia de cuya Seo era canónigo, al haber renunciado en 1504 al canonicato de Xàtiva.

En junio de 1505 disponía su testamento 6 en el que nombra por albaceas a Felipe Cardador, ciudadano, de la ciudad de Xàtiva, su padre y a Baltasar Ferran, caballero, su cuñado. Elige sepultura en la capilla que el reverendísimo señor don Francisco de Borja, cardenal y arzobispo de Cosença, su tío, ha obrado y edificado en la Seo de la ciudad de Xàtiva, en el vaso que está dentro de ella, en el cual estaban enterrados algunos de sus parientes. Quiere que se instituya un beneficio en dicha capilla y que sea dotado de 18 libras de renta, dejando como patrono del mismo a su padre Felipe Cardador.

Quiere que los dichos Felipe Cardador, y mosén Baltasar Ferran, y su señora madre y hermanas y todos los de la casa de su padre y suya se vistan de duelo, los hombres de gramalla y las mujeres de mantells. Nombra heredero a su padre, sólo durante la vida de éste y luego, a su muerte, será su

\footnotetext{
1 Viciana, Martín de, Crónica de la Inclita y Coronada ciudad de Valencia, Valencia, 1564, edición del Departamento de Historia del Arte Moderno, Valencia, 1972, Tercera parte, pp. 332-333.

2 Sobre los restos de la capilla y su lápida ver, González Baldovi, Mariano, "Les emprentes del mecenatge dels Borja a Xàtiva” Xàtiva, els Borja, una projecció europea, 1995, Tomo I, pp. 239-254.

${ }^{3}$ Estas aclaraciones sobre los difíciles entronques familiares en González Baldoví, Mariano, "Los Borja: Un linaje de Xàtiva para la historia" en Los Borja. Del mundo gótico al universo renacentista, Valencia, 2000-2001, pp. 65-77.

4 También se ha confundido su biografía y se le ha identificado con Francesc de Borja Navarro d'Alpicat, aplicando a este nombre sus datos biográficos en Garín, Felipe, "Mare de Déu de les Febres", Xàtiva, els Borja, una projecció europea, 1995, Tomo II, pp.183-185., y en Hinojosa, J., Diccionario medieval valenciano, Valencia, 2002, T. I., p. 377 ,

5 Aunque Tormo, Elías, en Un museo de primitivos. Las tablas de las iglesias de Játiva, creyó que era un solo obispo, esta confusión la aclararía Pascual, Ventura, en "Dos obispos de Teano, setabenses y homónimos" Boletín de la Real Academia de la Historia, Tomo LXXVI, cuaderno IV, abril 1920 y en Játiva Biográfica, Valencia, 1931, Tomo I, pp.288-294 y Tomo II, pp. 301-305

${ }^{6}$ Archivo Protocolos Patriarca de Valencia, en lo sucesivo APPV, notario: Guillem Ramon Tovià, sig: 26408.
} 
heredero dicho reverendo Francisco de Borja, hermano de su madre. Fueron testigos los venerables mosén Pere Çelles y mosén Pere Cerdá, presbíteros, beneficiados en la Seo de Valencia y Nofre Lop, músico.

En 1508 era nombrado obispo de Teano por renuncia a su favor que hacía su tío el cardenal Francisco de Borja. Sabemos que había comprado el señorío de Sent Pere pequeño lugar, con sus anejos Sella y Cartayna, junto a Benigánim, que en 1537 lo había cedido a su hijo Francisco de Borja y Cardador, caballero, comendador de Santiago ${ }^{7}$. Este hijo Francisco, tenido seguramente con su fiel sirvienta Isabel Cola ${ }^{8}$, estaba casado con Eleonor Juan con la que tuvo dos hijas, Hipólita, su heredera e Isabel. El 11 de junio de 1537, redactaba su testamento ante el notario Pasqual Perpinyá ${ }^{9}$, fundando un vínculo con sus bienes, que pasaría al extinguirse la rama de Hipólita a los descendientes del matrimonio formado por Isabel y Fernando Inse de San Juan ${ }^{10}$.

Francisco de Borja, al igual que su predecesor el cardenal Alfonso de Borja, quiso erigir esta capilla dedicada a la Virgen de las Fiebres en Xàtiva, ciudad en la que había nacido entre 1435 y 1440, y en la que residió muy poco tiempo, ya que vivió la mayor parte de su vida en Roma. Carecemos de datos sobre su construcción aunque es de presumir que en 1497 estaba muy avanzada y empieza a procederse a su decoración, que duraría hasta 1511, y que no vería concluida, pues el cardenal fallece en Italia en noviembre de ese año, enterrándose en Regio Lepido. Ya en fecha temprana, junio de 1505, hemos visto como su sobrino, Francesc de Borja Cardador entonces canónigo de la Seo de Valencia había dispuesto en su testamento su deseo de elegir sepultura en el vaso que había en la capilla del reverendísimo Francesc de Borja, cardenal y arzobispo de Cosenza, que su tío había obrado y edificado en la Seo de la ciudad de Xàtiva. A pesar de este temprano testamento, la muerte de Francesc de Borja Cardador no se produciría hasta 1540, cuando él había adquirido los derechos de sepultura por disposición del cabildo de 1515, ya que el cardenal había fallecido sin haber ordenado la sucesión en la titularidad del patronazgo. Pasaría luego a ser vulgarmente conocida como "capella del bisbe", al ser nombrado Francesc de Borja Cardador, segundo obispo de Teano, como hemos indicado. Otros miembros de la familia, la utilizarían con posterioridad como capilla funeraria.

La capilla se fue adornando con objetos procedentes de Italia, entre los cuales se ha destacado siempre la tabla de Pinturicchio con la Virgen de las Fiebres y el donante, el propio Francisco de Borja, enviada entre 1497 y 1499, antes de su nombramiento como cardenal, y que afortunadamente se ha conservado y se exhibe en el Museo de Bellas Artes de Valencia (fig. 3). También consta el envío de un relicario con dos puertas pintadas, una con la escena del Nacimiento de Cristo y la otra con la Pasión, en 1502, del cual tenemos noticia por una relación de las reliquias conservadas en la seo de Xàtiva de $1620^{11}$ y por un manuscrito de mediados del siglo XVII ${ }^{12}$.

Otro elemento de interés que presentaba esta capilla era una estructura a modo de pequeño baldaquino contratada el 3 de octubre de 1498 con el carpintero de la ciudad de Valencia, Carlos Gonzálbez ${ }^{13}$. Se denomina "scannum" en la documentación y presentaba cuatro columnas de

\footnotetext{
${ }^{7}$ El lugar de Sent Pere lo vende Hipólita de Borja el 20 de junio de 1607 a Jerónimo Nuñez y a su mujer Rafaela Bosch. Su hijo Pedro, señor de Sent Pere, se casaba en 1633 con Francisca Vallterra, hija de Bernabé Vallterra y de Rafaela Burgos.

8 Ver González Baldoví, Mariano, "Los Borja... opus cit.

${ }^{9}$ Este Francisco, quien seguramente premurió a su padre, se confunde con el obispo, al que se le atribuye este testamento. Por tanto son tres los Franciscos de Borja de esta familia.

${ }^{10}$ En sentencia dada por el Justicia Civil de Valencia el 9 de febrero de 1664 se concedía este vínculo a María Boil Borja Cardador y Malferit, mujer de José Vallterra Reyna, padres de Pedro, marqués de Vallterra.

11 González Baldoví, Mariano, "Les emprentes... opus cit.

12 Pascual, Ventura, Játiva biográfica... opus cit.

13 Sanchis Sivera, José, "Maestros de obras y lapicidas valencianos en la Edad Media" Archivo de Arte Valenciano, 1925, p. 51, revisado en Archivo del Reino de Valencia, en lo sucesivo ARV, notario: Jaume Prats, sig: 1863, 30 de octubre
} 
cinco palmos y dos tercios, poco más de 1'20 metros de altura y cuatro palmos de longitud ${ }^{14}$, o menos de 1 metro de anchura, y un cuadro para la parte superior de tres palmos de ancho y cuatro de alto. El intermediario en esta contratación es su sobrino Francisco de Cardador, entonces canónigo de Valencia, quien en 15 de diciembre de 1505 termina de pagar este trabajo. Esta palabra, "scannum" en documentos posteriores se emplea para definir un banco de altar o retablo, y quizá pueda ser esa su interpretación ${ }^{15}$. Carlos Gonzálbez ya había trabajado con anterioridad para la familia Borja, y por mediación de sus procuradores, el cardenal Rodrigo de Borja le había encargado el retablo que debía pintar con posterioridad Rodrigo de Osona para el transaltar de la catedral de Valencia ${ }^{16}$.

Previamente, y por mediación del mismo Francesc de Borja Cardador y del canónigo Joan Sanchis el 4 de abril de 1505 se había contratado con el mestre Joan Pons alias Aloy de Valencia, la reja para la capilla ${ }^{17}$. La reja debía ser como la de la capilla de mestre Climent ${ }^{18}$ de la catedral de Valencia, con arquillos y decoración de follajes, según el dibujo en manos de ambas partes. El 20 de noviembre del mismo año $1505^{19}$ se encargaba el flautado y "cimbol" de un órgano, al maestro y vecino de Valencia Lorenzo Jorba ${ }^{20}$ quien debía seguir el modelo del órgano del señor de Buñol.

\section{El contrato de los Hernandos para la capilla de les Febres}

El martes 25 de agosto de 1511 se firmó el contrato entre Guillermo Raymundo de Centelles canónigo de Valencia en calidad de procurador de Francisco de Borja, cardenal y arzobispo de Cosenza de una parte y los maestros Ferrando de Llanos y Ferrando de Medina, pintores de retablos, castellanos y residentes en aquel momento en la ciudad de Valencia, de otra sobre la pintura y pintar el retablo del señor cardenal que estaba en la capilla del cardenal de la Seo de la ciudad de Xàtiva ${ }^{21}$.

de 1498, contrato en latín con Karolus Goçalbez carpentario et magister operis talla in civitate Valencie de gens... quondam scannum quatuor palmorum cum dimidio latitudinis et quatuor columpnas latitudinis videlicet palmorum et duorum terciorum cum omnibus illius operibus in form als mostra e lo quadro dalt sent trium palmorum in dimidio latitudinis et quatuor palmorum cum dimidio longitudinis..."

${ }^{14}$ Las medidas se encuentran mejor especificadas en el ápoca de pago inédita de 1505, que es presumiblemente cuando se terminó de entregar este objeto, ARV, notario: Jaume Prats, sig: 1870, 15 de diciembre de 1505, "cuidam escani quatuor palmorum in dimidio latitudinis et quatuor columpnarum latitudinis videlicet cinque palmorum duorum terciorum...'

15 APPV, notario: Luis Baldó, sig: 4280, 15 de septiembre de 1560, contrato para la realización por parte del escultor de Xátiva, entonces habitante de Valencia, Jerónimo Muñoz, de un "scannum sive lo banch altaris sive retabuli", para la capilla de la Virgen de la Consolación en la iglesia parroquial de la Santa Cruz de Valencia.

16 Company, Ximo, El mundo de los Osona, Cat. Exp., 1994, p. 243, comprobado en el protocolo de APPV, notario: Andreu Cirera, sig: 20622, 10 de abril de 1483, es un ápoca de pago de 22 libras en parte de paga del total de 40 libras que paga Joan Vidal presbitero beneficiado del Cardenal, "pro facere quoddam retabulum quod pro reverendis Cardinalis mandat fieri retro altare maioris sedis Valencie" y otras 15 libras "per fer certa tuba e vasos que he fetes en lo dit retaule".

17 APPV, notario: notario: Guillem Ramon Tovià, sig: 26408, 14 de abril de 1505 .

18 La reja de la capilla de mestre Climent la doraba en 1492 el pintor Pere Cabanes, o sea que debía estar recientemente terminada, ver Sanchis Sivera, José, "Pintores medievales en Valencia”, Archivo de Arte Valenciano, 1930-31, p. 73.

${ }^{19}$ APPV, notario: notario: Guillem Ramon Tovià, sig: 26408, 20 de noviembre de 1505.

${ }^{20}$ Este maestro de órganos ya se había encargado junto a Joan Forment de realizar el órgano de la iglesia del monasterio de Cotalba en 1475, APPV, Mateu Cirera, sig: 21879, 27 de noviembre de 1475.

21 APPV, notario: notario: Guillem Ramon Tovià, sig: 26407, 25 de agosto de 1511, ver apéndice documental. 


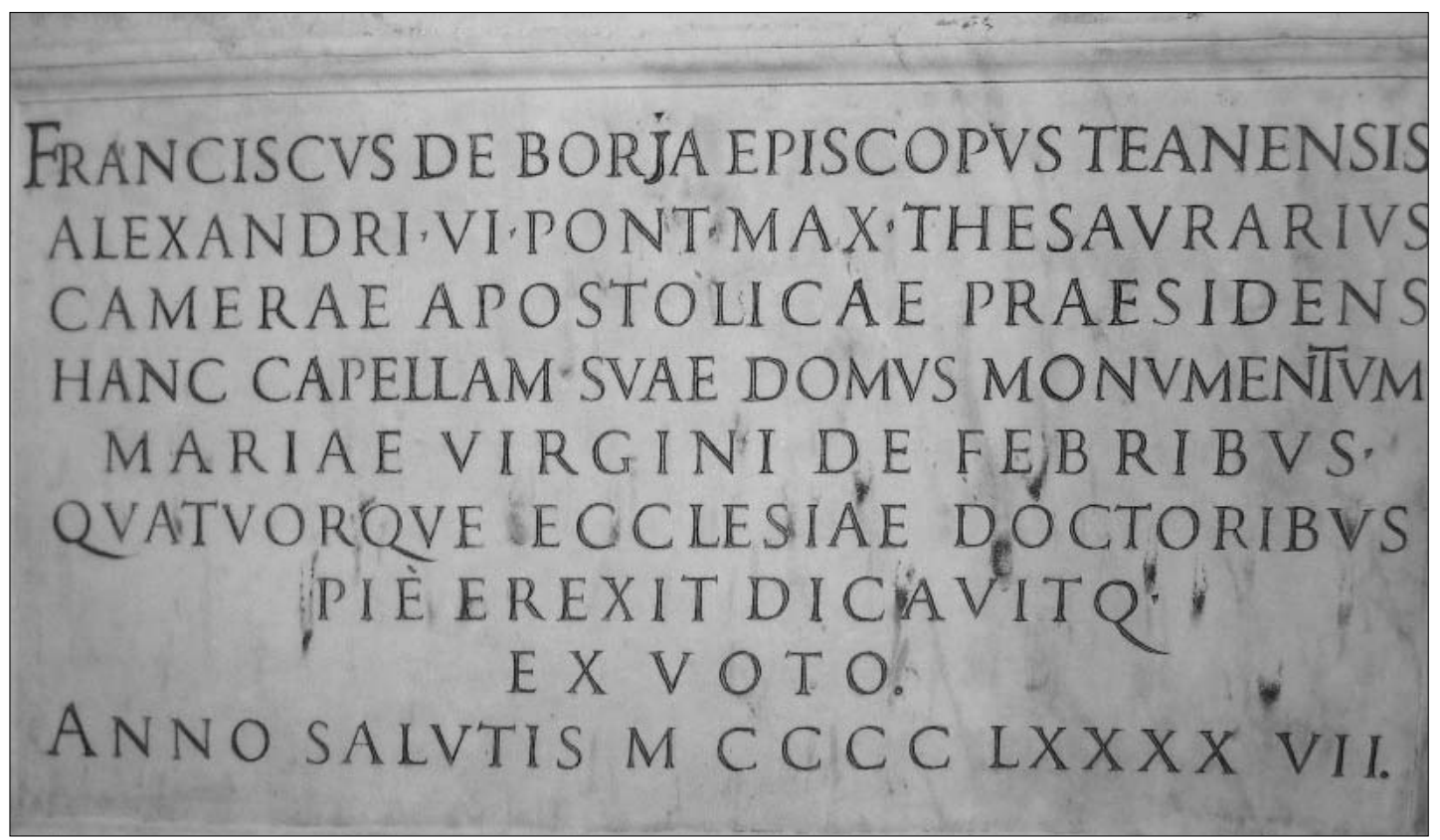

Fig. 1. Lápida conmemorativa de la fundación de la capilla de les Febres en la Colegiata de Xàtiva.

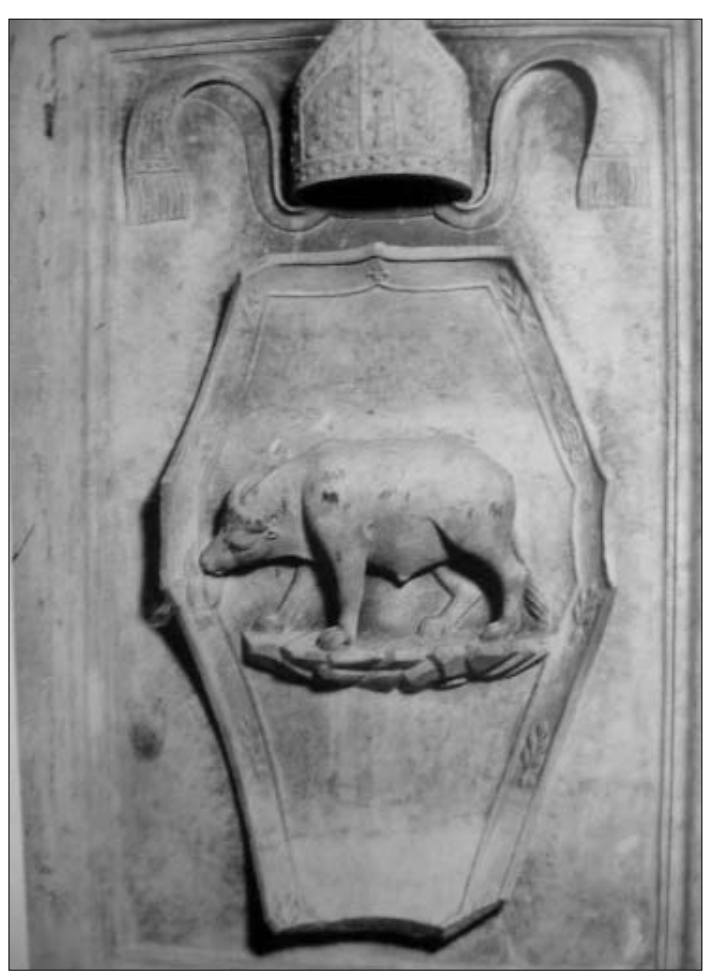

Fig. 2. Escudo del obispo don Francisco de Borja.

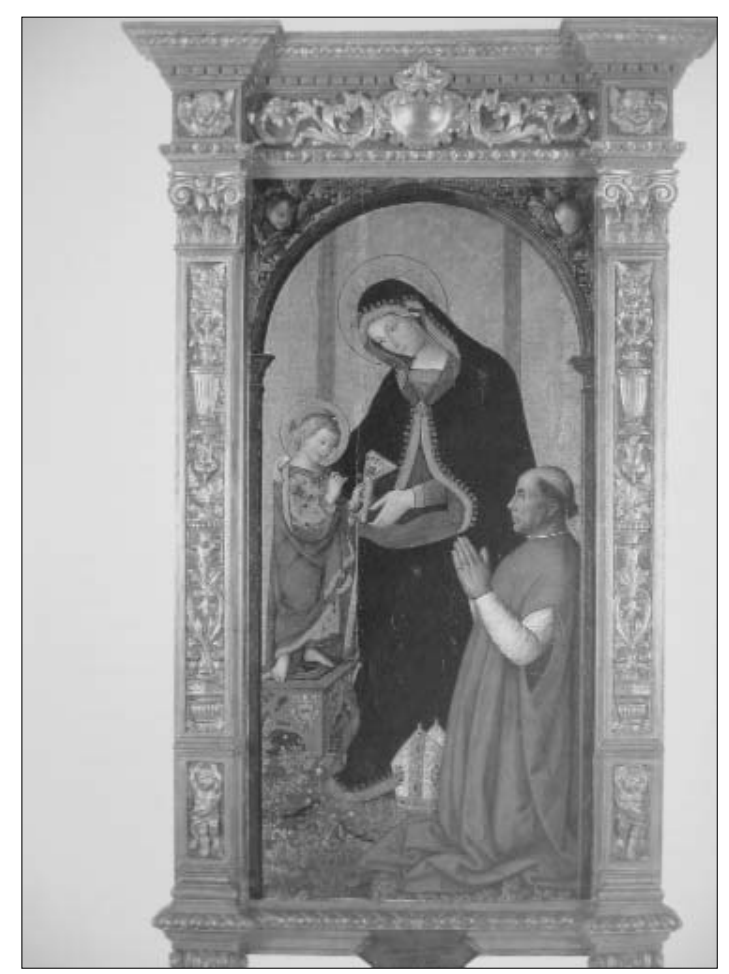

Fig. 3. Tabla de la Virgen de las Fiebres, de Pinturicchio, con el retrato del donante, don Francisco de Borja. 
Los capítulos del contrato no indican la temática del retablo, que al parecer estaba ya empezado, "quant ne resta per pintar e acabar com hi ha ara ja pintades certes peces e taules". Para las tablas y piezas que faltaban por pintar, debían ajustarse a las indicaciones de la hermana del cardenal, mujer del caballero Felipe Cardador, y del canónigo Centelles. Las historias se debían pintar al óleo, y también se debía pintar y dorar parte del retablo que estaba ya comenzado "a la romana". Todo esto debía hacerse en la ciudad de Xâtiva, a donde los pintores debían desplazarse el 10 de septiembre de 1511 y donde se les proporcionaría una casa. Por todo ello recibirían 150 ducados de oro, en tres pagas, la primera a su llegada a Xàtiva, la segunda cuando se comenzara a poner el color y la última con el retablo ya acabado, que debía ser en el mes de abril de 1512.

De estas capitulaciones se infieren una serie de datos importantes, que contribuyen a clarificar la controvertida trayectoria de los Hernandos, objeto de muchas especulaciones. El documento puede probar algunos hechos, aunque no todo son respuestas y seguimos ante muchos interrogantes, que no acaban de poder solucionarse.

Se trata de un documento significativo en la trayectoria de los Hernandos 22 que completa el conocimiento que tenemos de la obra realizada en el periodo valenciano. Pertenece a una época en la que como vemos, aún seguían formando una compañía, ya que el encargo es a los dos pintores juntos. Vuelve a aparecer citado en primer lugar Fernando de Llanos y en segundo Yáñez, a quien se denomina Fernando de Medina, y cuando se nombra a los dos juntos se hace como a "los dits mestres Ferrandos, pintors". Este aspecto del lugar en que se nombran los pintores en los documentos, había sido esgrimido para señalar la mayor edad o importancia de uno con respecto a otro. Parecía que las únicas veces en que Llanos era citado en primer lugar era en la documentación de las puertas del retablo de la catedral y sus pagos, y que luego ya nunca volvía a ser así, porque Yáñez a partir de esta obra adquirió una cierta preeminencia como demostraban los contratos y documentos posteriores relacionados con el retablo del gremio de plateros, y el encargo del órgano de la catedral. Nuevamente, vemos que en un documento posterior al pleito del gremio de plateros, 1509-1510, nos encontramos citado a Llanos en primer lugar. Luego, no podemos hacer de este aspecto, el orden de preeminencia en la documentación, un medio para distinguir la mayor importancia de uno o de otro. Este aspecto deja de ser tan determinante y no permite llegar a ninguna conclusión segura 23 .

En el contrato de Xàtiva se les conoce a ambos como pintores de retablos, castellanos, que de presente estaban viviendo en Valencia, aunque vemos que una de las claúsulas recoge la obligación de trasladarse y residir en Xàtiva para pintarlo. Es una prueba más del carácter itinerante de los pintores, que aunque fijaban la residencia en una población, podían trasladarse temporalmente a otra y regresar de nuevo a Valencia, una vez cumplido el contrato, como sucederá en fases ulteriores de su carrera. En este punto cabe señalar contrariamente a lo que se ha venido creyendo hasta ahora, que Llanos no se instaló definitivamente en Murcia en 1514 para no regresar más a Valencia, sino que sabemos que el 12 de mayo de 1517 estaba habitando sólo en Valencia, donde se cita como "Ernandus de Lanos, pictor retabulorum civitatis Valencie habitator", fecha en que nombra procuradores a Jacobo Sanç platero y a Nicolás Falcó, pintor ${ }^{24}$. Con lo que se abre un nuevo tema de investigación, el regreso de Llanos a Valencia, su relación con otros artistas,

\footnotetext{
${ }^{22}$ Esta trayectoria revisada en el Catálogo de la Exposición, Benito, Fernando, Los Hernandos, Valencia, 1998. Para un estudio muy completo, especialmente dedicado a Yáñez, pero que revisa toda la problemática y resume y recoge interesantes y novedosas aportaciones ver Ibáñez, Pedro Miguel, Fernando Yáñez de la Almedina. (La incógnita Yáñez), Cuenca, 1999.

${ }^{23}$ Ibáñez, Pedro Miguel, Fernando Yáñez... opus cit., p. 86 , indicaba que inicialmente era Llanos el que se mencionaba en primer lugar, y que parecía como a partir de 1509 fue ya Yáñez.

24 Ver Gómez-Ferrer, Mercedes, “Artistas viajeros entre Valencia e Italia, 1450-1550”, Saitabi, 50, Valencia, 2000, pp. 151-170.
} 
especialmente con Falcó, y otro controvertido asunto, la falta de relación con Yáñez, quizá ya por ausencia de éste de la ciudad, quizá por la definitiva disolución de la compañía. Llanos, no obstante, volvería a Murcia en 1520, esta vez parece que para quedarse.

El contrato de Xàtiva es además prueba de colaboración de los dos Hernandos fuera del ámbito catedralicio ${ }^{25}$, que se suma a la pintura y dorado de la imagen del retablo y cuatro historias de la predela del gremio de plateros en la iglesia de Santa Catalina de Valencia, concertada el 7 de febrero de 1509. Para este retablo no sólo pintaban las historias y doraban la imagen principal, sino que diseñaban la estructura de madera que debían ejecutar los Forment, Onofre y Damián, y que suscitó un intenso pleito con el gremio de plateros ${ }^{26}$ que también nos proporciona información sobre lo ejecutado por los Hernandos en estos primeros años de estancia en Valencia. Por tanto, queda claro que el ámbito de colaboración y formación de una compañía entre Llanos y Yáñez supera la mera asociación para la realización de las puertas del retablo de la catedral y otras actuaciones conjuntas en esas fechas, como la citada del retablo de plateros o la tasación de unas pinturas en la iglesia de Santo Tomás, para asumir otro gran compromiso conjunto como era un nuevo retablo en otra población. En esta fecha, agosto de 1511, aún no se habrían separado y no podemos concluir que Yáñez destacara por encima de su colega, acaparando ya encargos de forma individual. Esa fase queda para una etapa ulterior, al menos después de 1513 en que aún compartían casa y taller en la parroquia de San Andrés de Valencia.

El periodo de agosto de 1511 a abril de 1512, nos permite llenar un vacío, que existía en esta primera parte de su trayectoria profesional en Valencia, que es no obstante, la mejor documentada por las obras de la catedral: retablo de los Santos Médicos, puertas del altar mayor, trazas del órgano y pinturas de las espaldas del "organet chiquet". El último pago recibido por las puertas del retablo mayor de la catedral es de 18 de septiembre de 1510. Este periodo también es coincidente con la finalización del retablo del gremio de plateros que debía concluirse en el plazo de dos años desde que firmaron las capitulaciones (febrero de 1509), por tanto hacia febrero de 1511. El siguiente pago documentado es a Yáñez en calidad de autor de los diseños para el órgano de la catedral y ya se fecha en 8 de noviembre de 1512. Confirmamos la posibilidad de realización de esta obra ya que existe un vacío documental desde febrero de 1511 hasta noviembre de 1512, que bien se puede llenar con este contrato para Xàtiva. Al no aparecer documentado Yáñez en la catedral a partir de 1511, ya Sanchis Sivera había advertido la posibilidad de que se encontraran realizando una obra fuera de Valencia. ${ }^{27}$

No obstante, es necesario realizar algunas precisiones. El contrato se firma el 25 de agosto de 1511 y los pintores debían marchar a Xàtiva el 10 de septiembre de 1511, cuando se presupone que recibirían el primer pago, extremo que no hemos podido confirmar al encontrarse incompleto el protocolo donde se encuentra el contrato. El protocolo se termina el 13 de septiembre y hasta esa fecha no hay consignada ápoca de pago a los Hernandos. Además los siguientes protocolos tampoco se conservan, por lo que no podemos comprobar si el trabajo efectivamente se realizó. Esta duda nos surge si tenemos en cuenta que el cardenal Francisco de Borja fallece en noviembre de 1511, aunque presumiblemente había dejado todo dispuesto, como había ocurrido en tantas otras ocasiones, en que los albaceas testamentarios se encargan de cumplir los deseos del testador y como además se hacía por mediación de sus procuradores presentes en la ciudad de Xàtiva, no es tampoco preciso que se tuviera que revocar.

\footnotetext{
25 Ibáñez, Pedro Miguel, Yáñez... opus cit., p. 87 señalaba que en su opinión la única prueba conservada de colaboración de los dos tocayos fuera del ámbito catedralicio, son las tablas de san Damián y san Cosme.

26 Salas, Xavier de, "Escultores renacientes en el Levante español", Anales y Boletín de los Museos de Arte de Barcelona, 1943, pp. 35-87.

${ }^{27}$ Sanchis Sivera, José, "Pintores medievales... opus cit. p. 100.
} 
Fig. 4. Retablo del Juicio Final de la colegiata de Xàtiva, obra de Yáñez de la Almedina (desaparecido en 1936).

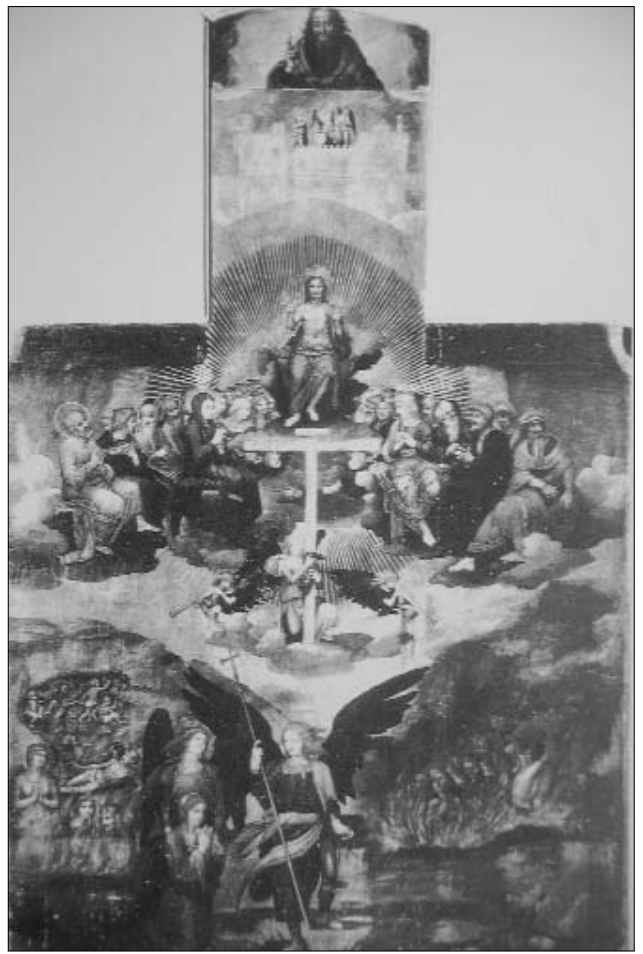

Estos procuradores pudieron seguir de cerca el proceso de terminación de la decoración de la capilla. Resulta significativa la mediación del canónigo Guillermo Raimundo de Centelles, uno de los canónigos presentes en el contrato del retablo mayor de la catedral de Valencia con los Hernandos, por tanto, persona cercana a ellos y que había seguido el proceso de la pintura del retablo mayor desde 1507. También la confianza en la hermana del cardenal y madre del obispo Cardador, quien junto a Centelles era la que propondría la temática de la obra y no de su hijo, quien había sido intermediario en los contratos anteriores de las obras de madera, rejas y órgano. Francisca, hermana del cardenal, siguió viviendo en Xàtiva y pudo estar perfectamente al tanto del cumplimiento de las obras pactadas, que por lo que imaginamos, estaban todas avanzadas (arquitectura, tabla de Pinturicchio, rejas, órgano y retablo comenzado)

La dificultad para la posible identificación de esta obra estriba en la falta de datos sobre su temática y a la pérdida del patrimonio pictórico de la ciudad de Xàtiva, que es especialmente complicada por dos motivos: el primero, la definitiva demolición de la antigua colegiata medieval en el último cuarto del siglo XVIII cuando se completan las obras que se habían iniciado a fines del siglo XVI, con el total desmantelamiento de las capillas existentes. A lo que se suma el grave incendio de 1936, que causó significativas pérdidas en su ya mermada colección.

De este importante patrimonio destaca por haber llegado hasta 1936, la importante tabla del Juicio Final de Yáñez, que conocemos por descripciones y por fotografía antigua (fig. 4). La perdida tabla del Juicio Final que perteneció a la Colegiata, generalmente se ha considerado obra de Yáñez en solitario, de un periodo tardío, para algunos entre 1515-1518, para otros entre 15191522. Esta fecha a su vez ha planteado no pocos problemas ya que en 1518 Yáñez regresa a su villa natal, Almedina, y no hay nueva documentación en Valencia, más que una referencia secundaria, aunque no desdeñable, de un platero de Valencia, Luis de Santafe, como depositario de los 
bienes del pintor en $1525^{28}$. Este dato sustenta la hipótesis de un regreso del pintor a Valencia tras las Germanías en 1523, que justifica lo que se ha denominado la producción tardía de Yáñez, entre la que se encuentra esta tabla del Juicio Final.

En realidad, este contrato tampoco arroja excesivas luces en la problemática sobre esta tabla. Sería una tentación demasiado fácil pretender una correlación entre la única tabla de uno de los Hernandos en Xàtiva y el único dato documentado de los Hernandos y Xàtiva. No parece que el retablo del Juicio Final de pala única pueda identificarse con esta obra de 1511, encargada a los Hernandos, ya que las claúsulas del contrato mencionan claramente varias tablas y algunas que ya estaban pintadas y debían completarse. Parece por tanto un retablo compartimentado, ya comenzado que tienen que terminar, caracterizado por su decoración "a la romana." En cierto sentido parecido a la obra que ejecutaron para el gremio de plateros en Santa Catalina. Otro problema es el de si al morir el mecenas que encarga la obra, se produjo una paralización del proyecto, o un cambio en la idea original, que retrasaría la ejecución, cambiaría la temática e incluso los autores. Aunque tampoco tiene mucho sentido relacionar una tabla de temática sobre el Juicio Final con una capilla dedicada a la Virgen de les Febres. Con lo que identificar esta obra del Juicio Final con una cronología más próxima a 1511 y a los dos maestros como autores de la misma, no haría más que complicar el análisis de la obra de Yáñez, y en el momento actual de las investigaciones y con el grado de conocimiento que tenemos de la misma, no haríamos más que entrar en un callejón sin salida. No parece muy sensato, a la vista de la escasa información que el documento proporciona sobre las características de la obra, pretender tal identificación.

De momento y con los datos que tenemos podemos hacer las siguientes consideraciones. Nos encontramos ante el nulo conocimiento sobre la temática exacta del retablo y sobre lo que ya había ejecutado antes del encargo a los Hernandos. La pérdida de los protocolos anteriores a éste, todos los comprendidos entre 1506 y 1511, no nos permite tampoco saber a quien se había encargado la obra con anterioridad, y si los Hernandos, sólo fueron autores de las pinturas que faltaban o también del diseño "a la romana" del mismo. El texto da a entender que también el armazón del retablo estaba ya empezado "a la romana" en el momento en que, según estos datos, los Hernandos entran en escena. Quizá su primera vinculación con la obra fuera la de este diseño y posteriormente se ampliara el contrato con la ejecución de algunas de las tablas. En este sentido conocemos que en 1510 aún era vecino de la ciudad de Xàtiva, y pagaba la tacha real el carpintero Luis Muñoz ${ }^{29}$, quien luego se trasladaría a Valencia, pues ya figura en julio de ese año como vecino de la ciudad en la declaración de los testigos de los Forment como consecuencia del pleito con los plateros, siendo a partir de 1512 uno de los principales escultores de las tallas del órgano de la catedral, trazadas por Yáñez. Quizá Xàtiva, y el retablo para el cardenal pudo ser un primer lugar de encuentro entre este escultor y Yáñez, que fructificaría luego en el órgano de la catedral.

No conocemos la temática y sólo sabemos que había ya algunas tablas pintadas. Se indica, además, que la pintura era al óleo, en las tablas; y aunque en primer término parece que el dorado sólo se refiera al armazón "han de dorar e pintar de colores ha hon sea menester en tot lo dit retaule segons ja esta començat a la romana", un último item nos indica que también las tablas pudieron tener dorados, "que sia pintat de colors fines, de or fi, millor ques fa e acostuma fer". En principio, se trataría de las sutiles aplicaciones de dorados que emplearon los Hernandos en algunas de sus tablas. Cenefas, orlas, motivos a candelieri y grutescos, nimbos, e incluso inscripciones, tienen pequeñas aplicaciones doradas.

28 Aportación de Ibáñez, Pedro Miguel, “Más allá de Valencia: el último Yáñez”, en Los Hernandos, Valencia, 1998, pp.127-142, que ha llevado a reconsiderar todo el periodo tardío de Yáñez.

29 Luis Muñoz figura como vecino de Xàtiva en la tacha real de 1510, ver Falomir, Miguel, Arte en Valencia, 1472 1522, Valencia, 1996, p. 498 y luego ya en Valencia en el pleito de plateros, ver Salas, Xavier de, "Escultores... opus cit. 
Considerando que actualmente no hay ninguna obra en Xàtiva que se pueda identificar claramente con este contrato, se nos plantea un dilema que nos conduce a varias probables hipótesis. Que el encargo de Francisco de Borja no llegara a ejecutarse y por tanto, no hubiera nada en Xàtiva que se pudiera relacionar con este documento. Que ante el fallecimiento de Francisco de Borja, el encargo se hubiera paralizado pero hubiera supuesto un contacto temprano de los Hernandos y Xàtiva, que se tradujera en fecha posterior en el definitivo encargo a Yáñez de la tabla del Juicio Final; una variante que modificaba el encargo inicial, si es que fue para esta capilla de les Febres, o la posibilidad de volver a contactar con uno de los Hernandos para otro encargo diferente en otra capilla de la colegiata, ya que desconocemos absolutamente todo sobre esta tabla. Que la obra de los Hernandos sí se llegara a realizar y fuera alguna de las obras perdidas de la colegiata de Xàtiva, como tantas y tantas otras de las que no nos ha llegado ni siquiera mención. O que pudiéramos tratar de identificarla con alguna de las mencionadas por los cronistas y estudiosos, que existieron hasta antes de 1936 en la colegiata.

Tenemos como referencias a la decoración de la capilla de les Febres, la donación de unas reliquias según consta por una relación de 1620, cerradas por dos tablas: "dos taules en les quals hi ha moltes imatges escolpides en vidre y dins aquelles, moltes reliquies de sants, les quals dona la bona memoria del cardenal de Cosenza". Estas se han tratado de identificar con un armario relicario con dos puertas, una con el Nacimiento y otra con la Pasión, según consta en un manuscrito con letra del siglo XVII que se encontraba en el convento de Predicadores, en 1931 y donde se indicaba que el cardenal envió desde Italia estas tablas y reliquias para el adorno de su capilla ${ }^{30}$. Ya en estas dos descripciones encontramos algunas indefiniciones como el hecho de que en la relación de 1620 se citan dos tablas con imágenes de vidrio, por lo que parece que puede ser un relicario con esmaltes o vidrios. Mientras que el otro texto describe como "Francisco de Borja Card. hizo la capilla de las Fiebres, impetró un breve de Alejandro VI para sacar reliquias de los cimenterios de Roma, asi del Pudenciano, San Jaime in Setignano como de otras partes, para inbiarlas a dicha capilla, su dato en 30 de septiembre de 1502, anno 11 de su pontificado. En fuerza dél hizo el Cardenal la diligencia y inbió dos tablas con sus niños (sic) para las reliquias. Esto es, una tabla donde estaba pintada la imagen de la Virgen y el Nacimiento de Xto., donde había las reliquias siguientes (...). La otra tabla tenía pintada la Pasión de Xpto y tenías las reliquias siguientes (...)" No hemos podido localizar el manuscrito original para tratar de interpretar la frase "dos tablas con sus niños" que creemos incorrecta. En cualquier caso parece deducirse que eran dos tablas pintadas y que ya fueron enviadas desde Italia.

Para tratar de establecer una posible relación entre este relicario y el retablo que nos ocupa nos desconcierta la mención de que sólo tenía dos tablas pintadas con el Nacimiento y la Piedad, y no varias como parece deducirse del contrato; aunque la descripción es tan tardía que bien pudiera estar ya incompleto, o simplemente no ser ésta la obra de los Hernandos. De todas formas, no sería la primera vez que los Hernandos trabajan en un armario relicario como fue el de los Santos Médicos de la catedral, primera de sus obras a la llegada a Valencia, que en la documentación se menciona como "retaule". No obstante, hay demasiadas contradicciones para tratar de identificar este armario relicario con la obra de los Hernandos. En primer lugar, el hecho de que se diga que las tablas habían sido enviadas desde Italia ya pintadas, y por otro lado, el que sean sólo dos, lo que parece asemejarlas a un armario, con dos puertas que se cierran, una con el Nacimiento y otra con la Pasión, y no a un retablo de varias tablas.

Otras descripciones de las obras artísticas que contenía la Colegiata antes del incendio de 1936 mencionan: "un tríptico de la Pietá, de lo mejor del prerrafaelismo itálico-valenciano acá traído por Pablo de San Leocadio, pintor enviado a Valencia por Alejandro VI, por 1472, vivo aún en

\footnotetext{
30 Sobre este tema González Baldoví, Mariano, "Les emprentes... opus cit.
} 
1501 en Gandía” en palabras de Tormo fechadas en $1912^{31}$, que en boca del cronista de Xàtiva, Ventura Pascual se convierten en "un tríptico de la Pietà, pintado en 1500 por un discípulo de Rodrigo de Osona, muy influenciado por el pintor de Alejandro VI, Pablo de San Leocadio, cuya influencia se nota más poderosamente en las escenas encantadoras de la vida de la Virgen que embelesan al cerrar las portezuelas y descubrirlas con asombro en la parte exterior de las mismas"32. Estos mismos autores, Tormo y Ventura Pascual sin embargo, identifican claramente la obra de Yáñez del Juicio Final como obra cumbre del renacimiento español, siguiendo lo que ya Bertaux había afirmado; por lo que es difícil pensar que se pueda confundir una obra de la escuela de San Leocadio con una de los Hernandos. Por la fotografía antigua publicada en el libro de Tormo y siguiendo su descripción, este tríptico era una obra escultórica con el tema de la Piedad, que se cerraba con unas puertas que en el exterior contenían escenas de la vida de la Virgen, y en el interior profetas y evangelistas, y que finalmente se considera obra de una misma mano relacionada con la escuela de San Leocadio. Estas tablas también se perdieron en el incendio de la Colegiata.

Por tanto, ninguna de las descripciones de las obras conservadas en la Seo antes de la guerra del 36 coinciden con un retablo atribuible a los Hernandos. Otra posibilidad, es que el retablo, se realizara pero quedara ya desmantelado cuando el traslado definitivo de la capilla original de las Fiebres a la nueva obra de la Colegiata en 1774, cuando la capilla se muda a instancias de D. Felipe Amorós y Darder de Borja, marqués de Sotelo y patrono de la capilla y sepultura de la capilla de las Fiebres ${ }^{33}$. Quizá aquí ya se pudo producir una primera dispersión de su patrimonio, que se completaría con el propio envío de la tabla de la Virgen de las Fiebres de Pinturicchio a Valencia en 1818, bien para servir como modelo a otra o para su restauración, según las dos versiones que se vienen barajando ${ }^{34}$. En este punto podríamos cuestionarnos sobre el papel de la tabla de Pinturicchio en la capilla. Si fue una tabla aislada o si fue tabla principal de un retablo que luego se pensaría completar. Por el momento, tampoco podemos concluir nada seguro al respecto.

Otra hipótesis es que las tablas encargadas a los Hernandos en 1511 para Xàtiva se pudieran identificar con algún conjunto concreto de los conservados y que pudiera en su día haber formado parte de un retablo. De estas características en el corpus conocido de los Hernandos sólo disponemos de las ocho tablas cuadradas, cuatro de las cuales se conservan en el Museo de Valencia, dos en colecciones particulares y dos desaparecidas, con temas de santos y de la Pasión, generalmente adjudicadas a Yáñez ${ }^{35}$. Se sabe que fueron reforzadas por un travesaño, donde está la fecha de 1817, un año anterior al traslado de la tabla de la Virgen de las Fiebres a Valencia, aunque no ingresaron en el Museo hasta después de la guerra civil. La elección de la temática se ha considerado característica de la catedral de Valencia, al incluir emparejados a San Antonino de Florencia y San Vicente Ferrer, ya que no hay constancia de esta doble advocación fuera de la catedral, y aunque se desconoce su procedencia, recientemente se han considerado relacionadas con las pinturas del "organet chiquet" de la catedral de Valencia pagadas a Yáñez en 1515. Esta iconografía de todas formas se puede explicar también fuera del ámbito catedralicio, si consideramos que uno de sus canónigos, Guillem Raymundo de Centelles fue el intermediario para la elección de los temas del retablo de los Hernandos en Xàtiva. Para Pedro Miguel Ibáñez ${ }^{36}$, la calidad de las tablas, la madurez de su estilo las

\footnotetext{
31 Tormo, Elías, Un museo de... opus cit., p. 14

32 Pascual, Ventura, El Turista en Játiva, 1925, p. 18. Descripción que repite Sarthou Carreres, Carlos, en Datos para la Historia de Játiva, tomo 1, p. 186.

33 Ver González Baldoví, Mariano, "Les emprentes... opus cit.

34 Company, Ximo,-Garín Llombart, Felipe, "Virgen de les Fiebres" en Madonas y Vírgenes, Cat. Exp. Valencia, 1995, pp. 138-143

35 Benito, Fernando, Los Hernandos, Valencia, 1998, pp. 182-197.

36 Ibáñez, Pedro Miguel, Yáñez... opus cit., p. 328-329.
} 
hace más bien piezas de un retablo que un mero trabajo ornamental, al tiempo que avanza su datación hacia 1510-11, y aunque adscritas a Yáñez, podrían entrar dentro de esta hipótesis, que no deja de ser una mera elucubración ya que nada las vincula con Xàtiva.

Podemos concluir que aunque nos hubiera gustado realizar una aportación más claramente concluyente al respecto de la obra de los Hernandos, el rigor que la investigación requiere ante la carencia de datos definitivos se suple con estas variantes e hipótesis que pretenden contribuir a enriquecer el difícil capítulo de la obra de Llanos y Yáñez. Creemos que los datos mostrados arrojan algunas luces, toda vez que animamos a los interesados en el tema a aportar opiniones acerca de esta problemática a raíz del documento y del análisis efectuado.

Apéndice documental. Archivo Protocolos Patriarca de Valencia, notario: Guillem Ramon Tovia, sig: 26407. 25 de agosto de 1511

Guillermus Raymundus Centelles canonge et prepositus sedis Valencie ut procurator reverendisimi domini Francisci de Borga Ex. Sr. et presbiter cardinalis et archiepiscopi Consentum ex una et Ferdinandus de Llanos et Ferdinandus de Medina pictori presens residentes partibus ex altera gratis et scienter in cum hoc presentis publico instrumento qonfitemur et in veritate recognoscimus notario ad invicem et vicisim quod fuerunt facta inhita concordata et in () ordinata subscripta capitula inter nos quod tenor sequitur sits hiis verba:

Capitols fets e fermats per e entre los reverent noble don Guillem Ramon Centelles canonge e paborde de la Seu de Valencia ex nomine e aixi com a procurador del reverendisimo don Francesc de Borga cardenal e archebisbe de Cosenza de una part e mestre Ferrando de Llanos e Ferrando de Medina pintors de retaules castellans residens de presents en la ciutat de Valencia de la part altra sobre la pintura e pintar un retaule del dit reverendisimo señor cardenal que esta en la capella del dit reverendisimo senyor cardenal en la Seu de la ciutat de Xativa.

E primerament es quonqordat e pactat entre les dites parts que los dits mestres Ferrandos sien tenguts pintar lo dit retaule de la capella del dit reverendisimo señor cardenal al oli de aquelles ystories o ymatges que sera necesari si e segon per la magnifica senyora germana del senyor excelentisimo cardenal muller del magnific Felip Cardador cavaller e per lo discret e reverent noble canonge Centelles per la altra de aquella los sera dit e donat memoria en la forma que volen lo dit retaule se pinte e acabar com hi ha ja pintades certes peces e taules.

Item es qonqordat e pactat entre les dites parts que los dits pintors han de daurar e pintar de colors ha hon sia menester en tot lo dit retaule segons sta ja començat a la romana.

Item es quonqordat e pactat entre les dites parts que lo dit retaule ha de esse pintat per los dits mestres en la ciutat de Xativa e no en altre part.

Item es qonqordat entre les dites parts que lo defahedor e assentar del dit retaule vingua tot lo que costara a despeses del Reverendissimo senyor cardenal.

Item es qonqordat entre les dites parts que lo dit retaule sia pintat de colors fines de or fi lo millor ques fa es acostume a fer es que les ymatges e ystories stiguen ab tota perfectio seguint la obra ja en dit retaule començada e millor si millor se pot fer.

Item es qonqordat e pactat entre les dites parts que lo dit reverent e noble don Guillem de Centelles en lo dit nom sia tengut e obligat donar e pagar a los dits mestres Ferrandos pintors per lo pintar y daurar per tot lo compliment del dit retaule cent cinquanta ducats de or los quals los hi ha de pagar en tres terçes e eguals pagues e terces, la primera paga $\mathrm{e}$ terça partint aquells de Valencia per anar a la dita ciutat de Xativa, la segona terça o paga haia de pagar quant se començara a obrar de colores la darrera quant ab tot compliment lo retaule e obra sia fet pintat e assentat.

Item mes es concordat e pactat entre les dites parts que los dits pintors haien de pintat ab tot compliment lo dit retaule per tot lo mes de abril del any vinent Mil D y dotze.

Item es qonqordat entre les dites parts que lo dit retaule ha de esser pintat de mans dels dits mestres Ferrandos pintors e no per mans de altre sots pena de cinquanta ducats.

Item es qonqordat entre les dites parts que los dits pintors han de esser presents en desarmar y armar del dit retaule fins que ab tot compliment stigua posat e asentat en la dita capella.

Item es qonqordat que lo dit reverent canonge Centelles lis donara o fara donar casa e habitacio convinent en la dita ciutat de Xativa per a estar e pintar lo dit retaule.

item mes es concordat entre les dites parts que [] partiran per anar a la dita ciutat de Xativa per a pintar lo dit retau-

le [] e [] X del mes de setembre en lo qual dia rebran la primera terça dels dits cent cinquanta ducats.

Quibus quidem (...) etc.

Testes Johannes e [] et discretus Joanes Splugues notari Valencia habitadors. 